\title{
EFFECT OF SCATTERING ON THE HEAT TRANSFER BEHAVIOR OF A TYPICAL SEMITRANSPARENT TBC MATERIAL ON A SUBSTRATE
}

Charles M. Spuckler

NASA Glenn Research Center

21000 Brookpark Rd.

Cleveland, $\mathrm{OH} 44135$

\begin{abstract}
This is a preprint or reprint of a paper presented at a conference. Since revisions may be made prior to formal publication, this version is made available with the understanding that it will not be cited or reproduced without the permission of the author.
\end{abstract}

\section{ABSTRACT}

A parametric study was performed to examine the effects of isotropic scattering on the heat transfer in typical semitransparent thermal barrier coating on an opaque substrate. Some ceramic materials are semitransparent in the wavelength range where thermal radiation is important. Therefore, absorption, emission, and scattering of thermal radiation by the semitransparent layer and the emissivity of the substrate will affect the heat transfer and temperature in the layer. Scattering which depends on the structure of the semitransparent material can be used to increase the reflectivity of the layer and therefore decrease the heat transfer through the layer. A one dimensional model of a $1 \mathrm{~mm}$ thick semitransparent layer on a substrate was used in this study. The front of the semitransparent layer is heated by convection and radiation and the back of the substrate is cooled by radiation and convection. The coating is assumed to be semitransparent up to a cutoff wavelength and opaque at higher wavelengths. The absorption and scattering coefficient are constant over the wavelength range where the material is semitransparent. The absorption coefficient, scattering coefficients, width of the semitransparent band, and the bond coat emissivity are varied. Temperature profiles and heat flux through layers as a function of absorption and scattering coefficients are presented.

\section{INTRODUCTION}

Thermal barrier coatings (TBCs) are being developed for use in gas turbine engines. TBCs can be made more effective by decreasing the heat conducted and/or radiated through them. Some thermal barrier coatings are partially transparent to thermal radiation. For example, for thermal radiation purposes zirconia can be semitransparent up to around $5 \mu \mathrm{m}$ (refs. 1 and 2). In semitransparent materials, both thermal radiation and heat conduction determine the temperatures and the heat transferred. Scattering, absorption, emission, and the refractive index determine the radiative heat transfer in a semitransparent material. The external and internal reflection of an interface between two semitransparent materials depends on the refractive index of the materials on each side of the interface. If thermal radiation is going from a material with a higher refractive index to one with a lower refractive index, there is a total reflection of the radiation at angles greater than the critical angle. Also, the thermal radiation emitted internally and by an opaque substrate into a material depends on the square of the refractive index. The internal thermal radiation passing through the semitransparent interface is decreased by internal surface reflections, which includes total internal reflection, so the energy emitted by the semitransparent layer can not exceed that of a blackbody. The refractive index can have a considerable effect on the temperature profile in a semitransparent layer.

The scattering and absorption coefficients determine the amount of thermal radiation absorbed, emitted, and scattered. These coefficients have units of reciprocal length. The reciprocal of the coefficients can be considered as the mean distance traveled before absorption 
or scattering occurs (ref. 3 page 424). The smaller the coefficient the larger the distance thermal radiation will travel before being absorbed or scattered. When thermal radiation is absorbed or emitted by a material its temperature changes. Absorption and emission therefore have a direct effect on the temperature of a material. Scattered thermal radiation has no effect on the temperature of a material unless it is absorbed. Scattering in some cases can act as additional absorption in determining the temperature profiles in a material ref. 4.

Increasing the scattering of a $\mathrm{TBC}$ is being considered as a method to improve their performance. A parametric study was performed to determine the effect isotropic scattering and absorption has on the heat flux and the temperatures in a ceramic material on a substrate. An absorption coefficient of $\mathrm{a}=0.1346 \mathrm{~cm}^{-1}$ and a scattering coefficient of $\sigma_{\mathrm{s}}=94.38 \mathrm{~cm}^{-1}$ were used as a base line. These coefficients are in the range of those of zirconia in wavelengths where it is semitransparent ref. 2. Because scattering depends on the material structure and the absorption is affected by impurities and temperature, the absorption and scattering coefficients are increased and decreased from the base line. Since the wavelength range over which some ceramic material are semitransparent decreases with temperature ref. 5 two cutoff wavelengths $5 \mu \mathrm{m}$ and $3 \mu \mathrm{m}$ were used. An infinitely thin bond coat was assumed, and the effect of the bond coat emissivity, $\varepsilon_{\mathrm{bc}}$, is shown by changing it from 0.3 to 0.7 .

\section{MODEL}

The model used, figure 1, is a semi-infinite semitransparent layer on a substrate. There is

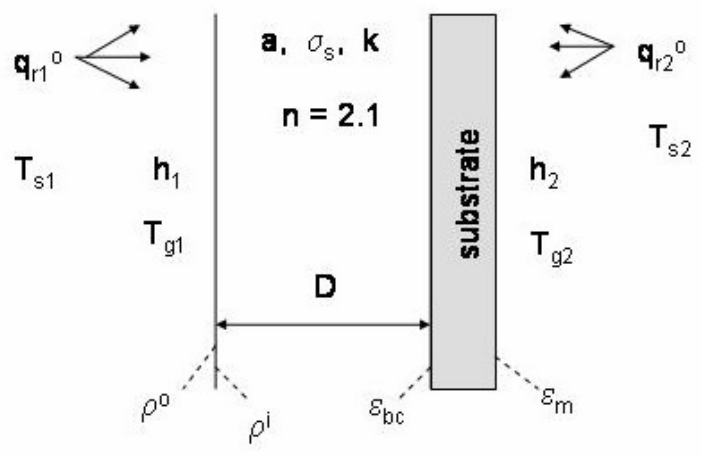

Figure 1 Heat transfer model

diffuse radiative and convective heat transfer on each side. The external radiative heating is $\mathrm{q}_{\mathrm{r} 1}{ }^{\circ}$ and $\mathrm{q}_{\mathrm{r} 2}{ }^{\circ}$. The hot side gas and surrounding temperatures, $\mathrm{T}_{\mathrm{s} 1}$ and $\mathrm{T}_{\mathrm{g} 1}$, are $2000 \mathrm{~K}$ and the cold side temperatures $\mathrm{T}_{\mathrm{s} 2}$ and $\mathrm{T}_{\mathrm{g} 2}$ are $800 \mathrm{~K}$. The $\mathrm{TBC}$ is $1 \mathrm{~mm}$ thick and has a thermal conductivity $\mathrm{k}=0.8 \mathrm{w} / \mathrm{mK}$. The substrate is $0.794 \mathrm{~mm}$ thick and has a thermal conductivity of $33 \mathrm{w} / \mathrm{mK}$. The heat transfer coefficients are $h_{1}=250 \mathrm{w} / \mathrm{m}^{2} \mathrm{~K}$ on the hot side and $\mathrm{h}_{2}=110 \mathrm{w} / \mathrm{m}^{2} \mathrm{~K}$ on the cold side. The emissivity of the back side of the metal substrate, $\varepsilon_{\mathrm{m}}$, is 0.6 . These conditions were used by Siegel (ref. 6) to determine internal radiation effects in a zirconia based TBC. The refractive index, $\mathrm{n}$, of the semitransparent layer is 2.1. The refractive index of the gas is assumed to be one. The external surface reflection, $\rho^{\circ}$, was calculated using Fresnel's equation for a nonabsorbing layer. This assumption should be good for the absorption coefficients used here (refs. 7 and 3 page 88). The internal surface reflection, $\rho^{i}$, was determined from a relationship using the refractive index and external surface reflection in ref. 8. A two flux approximation to the radaitive transfer equation was used to calculate the heat flux and the temperature profiles. The 
boundary conditions and the two flux equations in ref. 9 were modified to account for the nongray semitransparent layer and substrate.

\section{EFFECT OF ABSORPTION AND SCATTERING}

Interface temperatures and heat flux

The effects of isotropic scattering and absorption on the gas-TBC and the TBC-substrate interface temperatures are shown in figure 2. Below $5 \mu \mathrm{m}$ the material is semitransparent and

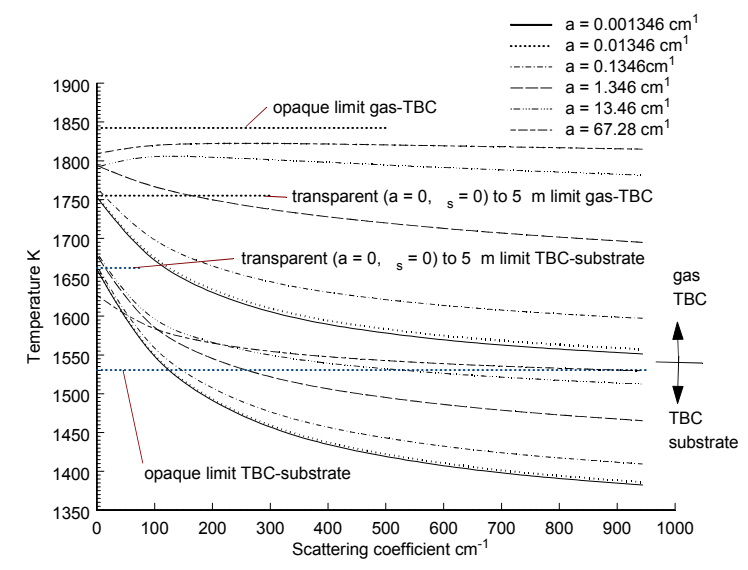

Figure 2 Effect of absorption and scattering on the interface temperature for $\varepsilon_{\mathrm{bc}}=0.3$ and $\lambda_{\mathrm{c}}=5 \mu \mathrm{m}$

above $5 \mu \mathrm{m}$ it is opaque. The emissivity of the bond coat is 0.3 . In general, the gas-TBC interface temperatures increase with absorption. The TBC-substrate interface temperature increases with absorption for all but the highest absorption coefficient used where the temperature decreased with absorption for lower scattering. Both the gas-TBC and TBCsubstrate interface temperatures decrease with scattering except for the gas-TBC interface temperatures for the two highest absorptions, which first increase and then decrease with scattering. The gas-TBC and TBC-substrate interface temperatures for the limiting cases of an opaque TBC $(\mathrm{a}=\infty)$ and a transparent TBC $\left(\mathrm{a}=0\right.$ and $\left.\sigma_{\mathrm{s}}=0\right)$ to $5 \mu \mathrm{m}$ are shown in the figure. The gas-TBC interface temperatures are less than the opaque limit, but can be greater than, equal to, or less than the transparent limit depending on the absorption and scattering. The TBCsubstrate interface temperatures are less than the transparent limit except for very low scattering. Depending on the scattering and absorption the TBC-substrate temperature can be greater than the opaque limit. Only for an absorption coefficient of $67.78 \mathrm{~cm}^{-1}$ are the temperatures less the transparent limit and about equal to or greater than the opaque limit. At this high of an absorption coefficient the TBC-substrate interface temperatures are starting toward the opaque limit.

The profiles for the heat flux in figure 3 are similar to the TBC-substrate profiles in figure 2. The heat flux decreases with scattering and increases with absorption except for the highest absorption, $67.28 \mathrm{~cm}^{-1}$ at lower scattering where the heat flux decreased with absorption. The limiting cases for the heat flux in a transparent $\left(\mathrm{a}=0\right.$ and $\left.\sigma_{\mathrm{s}}=0\right)$ to $5 \mu \mathrm{m}$ and opaque TBC $(\mathrm{a}=$ $\infty)$ are presented. For some absorption coefficients, 1.346 and $13.46 \mathrm{~cm}^{-1}$ and low scattering the heat flux is greater than for a layer that is transparent to $5 \mu \mathrm{m}$. For low and very high absorption the heat flux through the layer is less than a transparent layer. Depending on the scattering and 


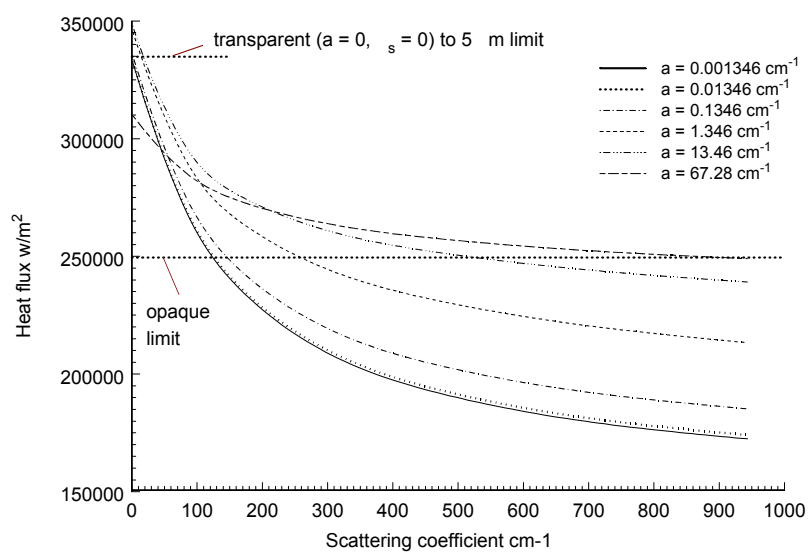

Figure 3 Effect of absorption and scattering on the heat flux for $\varepsilon_{\mathrm{bc}}=0.3$ and $\lambda_{\mathrm{c}}=5 \mu \mathrm{m}$

absorption the heat flux can be greater than the opaque limit. Only for the highest absorption coefficient, $67.28 \mathrm{~cm}^{-1}$, is heat flux greater than or about equal to that of an opaque layer. There is a significant decrease in the heat flux through the layer for low absorption and high scattering.

When the emissivity of the bond coat was increased to 0.7 and the cutoff wavelength maintained at $5 \mu \mathrm{m}$, the gas-TBC and the TBC-substrate interface temperature profiles as a function of scattering had trends similar to those in figure 2. The difference in the temperatures of gas-TBC interface for a bond coat emissivity of 0.7 and $0.3\left(\mathrm{~T}_{\varepsilon b c=0.7}-\mathrm{T}_{\varepsilon b c=0.3}\right)$ is given in figure

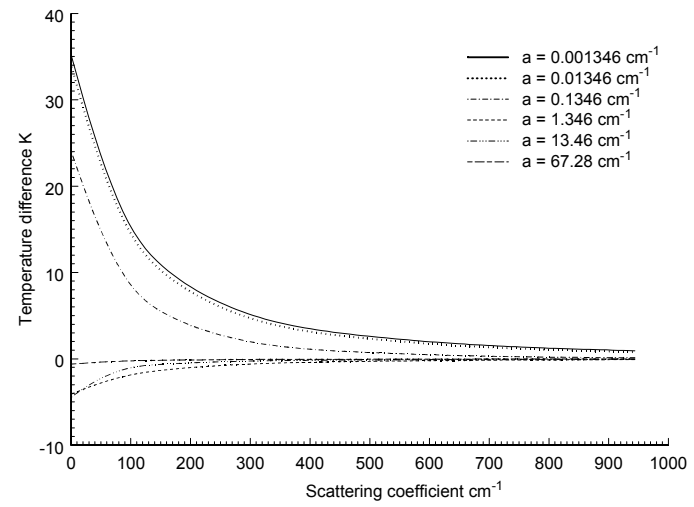

Figure 4 Difference in gas-TBC interface temperature for bond coat emissivity of 0.7 and $0.3\left(T \varepsilon_{\mathrm{bc}}=0.7-\mathrm{T} \varepsilon_{\mathrm{bc}}=0.3\right)$

4. For the three lower absorption coefficients the gas-TBC interface temperatures were higher for a bond coat emissivity of 0.7 . For the three highest absorption coefficients the gas-TBC interface temperatures were slightly lower for a bond coat emissivity of 0.7 . The temperature differences were greater at low scattering and decreased as scattering increased. At high scattering there was nearly no temperature difference. The effect of changing the emissitivity of the bond coat on the TBC-substrate interface temperatures $\left(T_{\varepsilon b c=0.7}-T_{\varepsilon b c=0.3}\right)$ is in figure 5. The TBC-substrate temperature for an emissivity of 0.7 is always greater than the TBC-substrate 


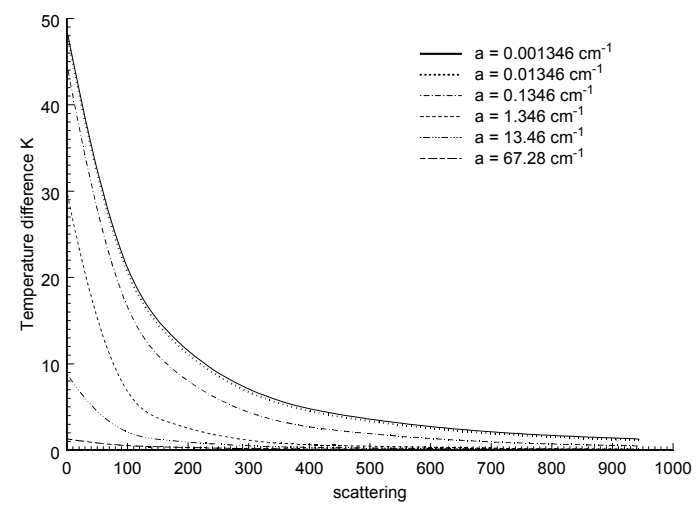

Figure 5 Difference in TBC-substrate interface temperatures for substrate emissivity of 0.7 and $0.3\left(T \varepsilon_{b c}=0.7-T \varepsilon_{b c}=0.3\right)$

temperature for an emisssivity of 0.3. The temperature difference decreased as either the scattering or the absorption increased. At low scattering and low absorption thermal radiation is able to penetrate to the substrate more easily and the effect of the bond coat emissivity is greater.

When the cutoff wavelength was reduced to $3 \mu \mathrm{m}$ and the substrate emissivity was 0.3 , the interface temperature profiles as a function of scattering again had the trends similar to

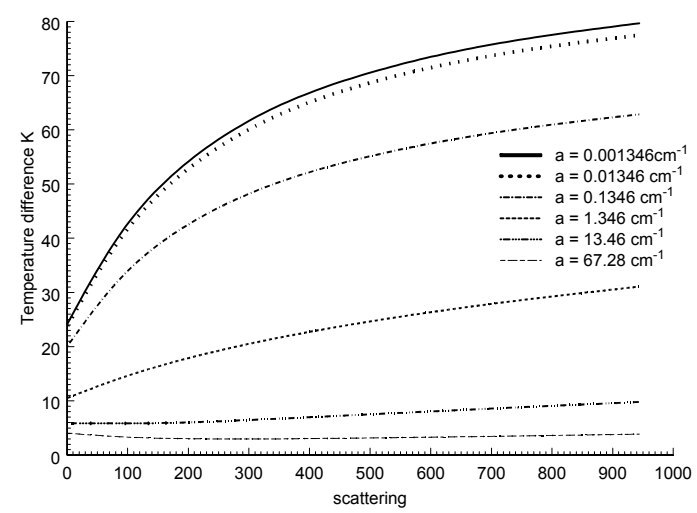

Figure 6 Difference in gas-TBC interface temperatures for cutoff wavelengths of $3 \mu \mathrm{m}$ and $5 \mu \mathrm{m}\left(\mathrm{T}_{\lambda=3 \mu \mathrm{m}}-\mathrm{T}_{\lambda=5 \mu \mathrm{m}}\right)$

those for a cutoff wavelength of $5 \mu \mathrm{m}$ (figure 2). The difference in the gas-TBC interface temperatures for cutoff wavelengths of $3 \mu \mathrm{m}$ and $5 \mu \mathrm{m}\left(\mathrm{T}_{\lambda=3 \mu \mathrm{m}}-\mathrm{T}_{\lambda=5 \mu \mathrm{m}}\right)$ is in figure 6 . The gasTBC interface temperatures for a cutoff wavelength of $3 \mu \mathrm{m}$ are always greater than the temperatures for a cutoff wavelength of $5 \mu \mathrm{m}$. The temperature difference increased with scattering and decreased with absorption except for the highest absorption where it at first decreased with scattering and then increased. The effect of scattering decreases as absorption increases. The difference in the TBC-substrate interface temperature for cutoff wavelengths of 3 and $5 \mu \mathrm{m}\left(\mathrm{T}_{\lambda=3 \mu \mathrm{m}}-\mathrm{T}_{\lambda=5 \mu \mathrm{m}}\right)$ is in figure 7 . Depending on the absorption and scattering the TBCsubstrate interface temperatures can be lower for a cutoff frequency of $3 \mu \mathrm{m}$ than for a cutoff frequency of $5 \mu \mathrm{m}$. The temperature difference $\left(\mathrm{T}_{\lambda=3 \mu \mathrm{m}}-\mathrm{T}_{\lambda=5 \mu \mathrm{m}}\right)$ either decreased or increased depending on the scattering and absorption. 


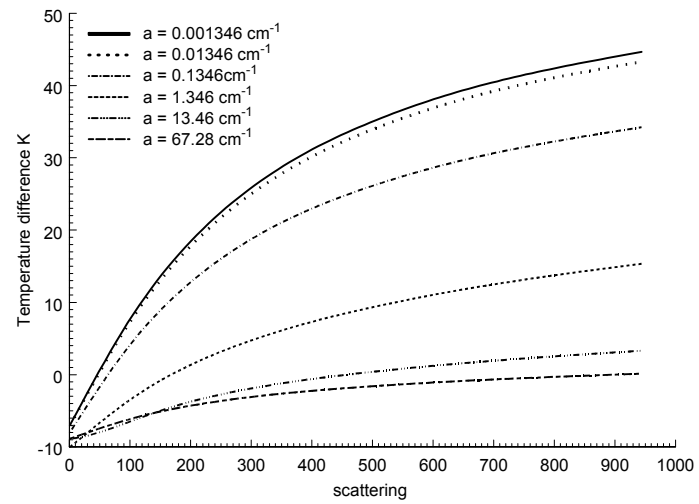

Figure 7 Difference in TBC-substrate interface temperatures for cutoff wavelengths of $3 \mu \mathrm{m}$ and $5 \mu \mathrm{m}\left(\mathrm{T}_{\lambda=3 \mu \mathrm{m}}-\mathrm{T}_{\lambda=5 \mu \mathrm{m}}\right)$

Internal temperature profiles

The temperature profiles in the TBC and substrate with low absorption coefficient $\mathrm{a}=$ $0.001346 \mathrm{~cm}^{-1}$ are shown in figure 8 . The opaque $(\mathrm{a}=\infty)$ and transparent $\left(\mathrm{a}=0\right.$ and $\left.\sigma_{\mathrm{s}}=0\right)$ to 5

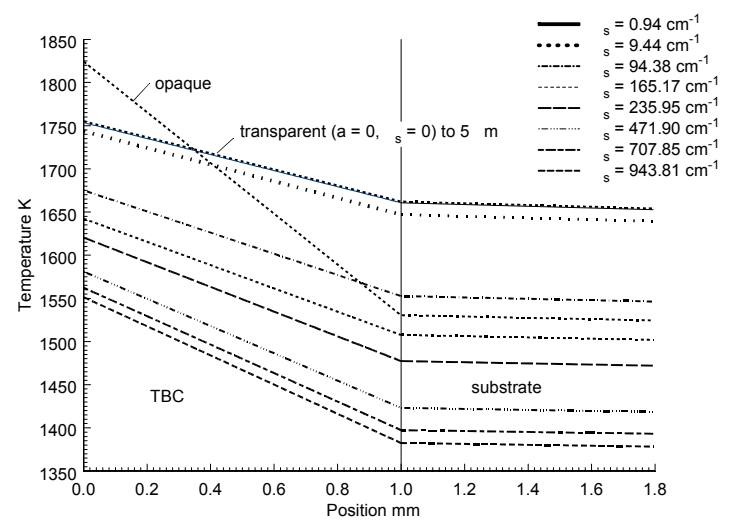

Figure 8 Temperatures in TBC and substrate as a function of scattering for $\mathrm{a}=0.001346 \mathrm{~cm}^{-1}, \lambda_{\mathrm{c}}=5 \mu \mathrm{m}$, and $\varepsilon_{\mathrm{bc}}=0.3$

$\mu \mathrm{m}$ limits are shown. The temperature profiles for the transparent limit and $\sigma_{\mathrm{s}}=0.94 \mathrm{~cm}^{-1}$ nearly coincide. The temperature profiles in the TBC are quite linear, indicating conduction is the dominate mode of heat transfer. The temperatures decrease as the scattering increases. As the scattering is increased with the absorption being low, more radiant energy is reflected by scattering sites in the $\mathrm{TBC}$, decreasing the radiant energy reaching the substrate. This causes a reduction in temperature in the $\mathrm{TBC}$ and substrate. The $\mathrm{TBC}$ and substrate temperatures are always equal to or less than the transparent limit. The gas-TBC interface temperature is considerably less than the opaque temperature limit. It is not until the scattering coefficient is about $125 \mathrm{~cm}^{-1}$ (see fig. 2 for more details), that the substrate temperatures are less than the opaque limit temperature.

The absorption coefficient is increased to $1.346 \mathrm{~cm}^{-1}$ in figure 9 . The temperature profiles have some curvature which indicates radiation is playing a role in the heat transfer process. For low scattering, some of the temperatures in the $\mathrm{TBC}$ and substrate are higher than the transparent 


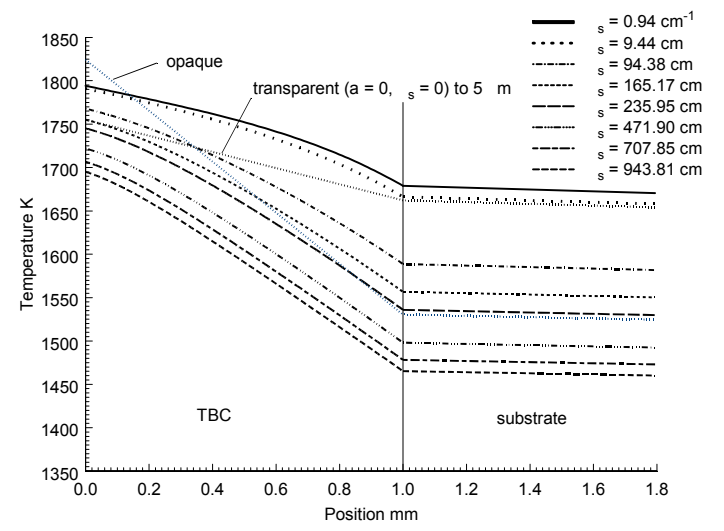

Figure 9 Temperatures in the TBC and substrate as a function of scattering for $\mathrm{a}=1.346 \mathrm{~cm}^{-1}, \lambda_{\mathrm{c}}=5 \mu \mathrm{m}$, and $\varepsilon_{\mathrm{bc}}=0.3$

limit. Now the scattering coefficient has to be greater than approximately $260 \mathrm{~cm}^{-1}$ (see fig. 2 for more details), for the substrate temperature to be less than the opaque limit.

The absorption coefficient is increased to $67.28 \mathrm{~cm}^{-1}$ in figure 10 . The gas-TBC interface temperatures for all scattering are greater than the transparent $\left(\mathrm{a}=0\right.$ and $\left.\sigma_{\mathrm{s}}=0\right)$ limit and less

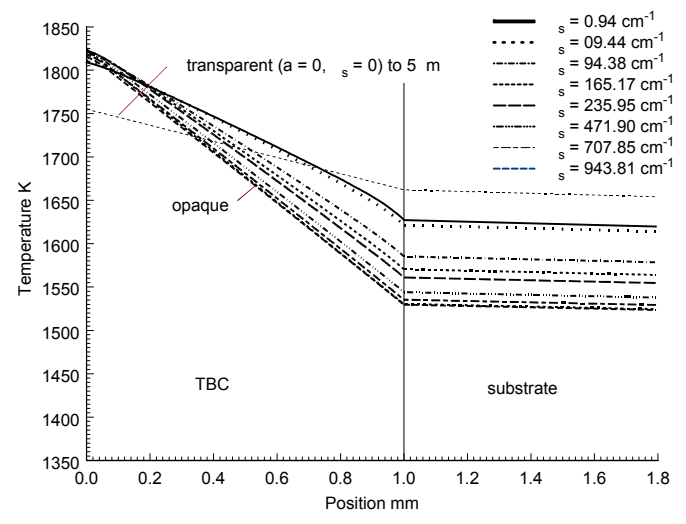

Figure 10 Temperatures in TBC and substrate as a function of scattering for $\mathrm{a}=68.27 \mathrm{~cm}^{-1}, \lambda_{\mathrm{c}}=5 \mu \mathrm{m}$, and $\varepsilon_{\mathrm{bc}}=0.3$

than but within $16 \mathrm{~K}$ of the opaque limit $(\mathrm{a}=\infty)$. The lowest gas interface temperature is for $\sigma_{\mathrm{s}}=$ $0.94 \mathrm{~cm}^{-1}$. The gas-TBC interface temperatures are approaching each other. The substrate temperatures are about equal to or greater than the opaque temperature limit.

\section{SUMMARY AND CONCLUSIONS}

A parametric study was performed to determine the effects isotropic scattering and absorption have on the temperatures and heat flux in a non-gray semitransparent layer on a substrate. A one-dimensional model was used. The TBC is $1 \mathrm{~mm}$ thick and the substrate is $0.794 \mathrm{~mm}$ thick. There is radiative and convective heat transfer on each side of the layers. The absorption and scattering coefficient were varied. The emissivity of the substrate and width of the wavelength band over which the material is semitransparent were changed. For the base case, cutoff wavelength of $5 \mu \mathrm{m}$ and a substrate emissivity of 0.3 , the gas-TBC interface temperature in general increased with absorption and for most cases decreased with scattering. The gas-TBC interface temperatures were always less than the opaque limit. Both the TBC- 
substrate temperatures and the heat flux through the layer decreased with scattering and increased with absorption except for high absorption and low scattering where they decreased with absorption. Depending on the scattering and absorption the substrate temperature and heat flux can be greater than the opaque limit. Therefore under some conditions an opaque TBC is more effective in reducing the substrate temperature and the heat flux. The effects of increasing the substrate emissivity from 0.3 to 0.7 were greatest at low scattering. The gas-TBC and TBCsubstrate interface temperatures where higher for an emissivity of 0.7 , except for high absorption where the gas-TBC interface temperatures were slightly lower for a 0.7 emissivity. In general, this temperature difference decreased as the absorption increased. The effects of decreasing the cutoff wavelength from $5 \mu \mathrm{m}$ to $3 \mu \mathrm{m}$ were greater for high scattering. The gas-TBC interface temperatures were higher for a cutoff wavelength of 3.0. Depending on the scattering and absorption the substrate temperatures can be lower for a cutoff wavelength of $3 \mu \mathrm{m}$ than $5 \mu \mathrm{m}$. For low absorption it seems that conduction in the TBC is the dominate mode of heat transfer. This study indicates scattering can be used to reduce the heat flux and the substrate temperatures for a $1 \mathrm{~mm}$ thick semitransparent layer especially for low absorption. For high absorption and low scattering an opaque layer may give better results than a semitransparent layer. A similar study for a thin semitransparent layer should be performed to determine the effects of thickness.

\section{ACKNOWLEDGEMENTS}

This work was supported by NASA Glenn Research Center Ultra-Efficient Engine Technology Program

\section{REFERENCES}

${ }^{1}$ Wahiduzzaman, $\mathrm{S}$ and Morel,T., Effect of Translucence of Engineering Ceramics on Heat Transfer in Diesel Engines, ORNL/Sub/88-22042/2, April 1992

${ }^{2}$ Makino, T., Kunitomo, T., Sakai, I., and Kinoshita, H., Thermal Radiation Properties of Ceramic Materials, Heat Transfer-Japanese Research, 13, [4] 33-50 (1984)

${ }^{3}$ Siegel, R. and Howell, J. R. Thermal Radiation Heat Transfer, $4{ }^{\text {th }}$ ed. Taylor \& Frances, New York, 2002

${ }^{4}$ Spuckler, C. M. and Siegel, R., "Refractive Index and Scattering Effects on Radiative Behavior of a Semitransparent Layer," Journal of Thermophysics and Heat Transfer, 7[2], 30210 (1993)

${ }^{5}$ Cabannes, F. and Billard, D. "Measurement of Infrared Absorption of Some Oxides in Connection with Radiative Transfer in Porous and Fibrous Materials," International Journal of Thermophysics, 8[1] 97-118 (1987)

${ }^{6}$ Siegel, R. "Internal Radiation Effects in Zirconia Thermal Barrier Coatings," Journal of Thermophysics and Heat Transfer, 10[4], 707-9 (1996)

${ }^{7}$ Cox, R. L., "Fundamentals of Thermal Radiation in Ceramic Materials,"; pp. 83-101 in Symposium on Thermal Radiation of Solids, edited by S. Katzoff, NASA SP-55, 1965

${ }^{8}$ Richmond, J. C., "Relation of Emittance to Other Optical Properties," Journal of Research of the National Bureau of Standards-C. Engineering and Instrumentation, 67C [3], 217-26 (1963)

${ }^{9}$ Siegel, R. and Spuckler, C. M., "Approximate Solution Methods for Spectral Radiative Transfer in High Refractive Index Layers," International Journal of Heat and Mass Transfer, 37 [Suppl. 1] 403-13 (1994) 